\title{
LA CORRUPCIÓN: UN PROBLEMA DE HUMANIDAD*
}

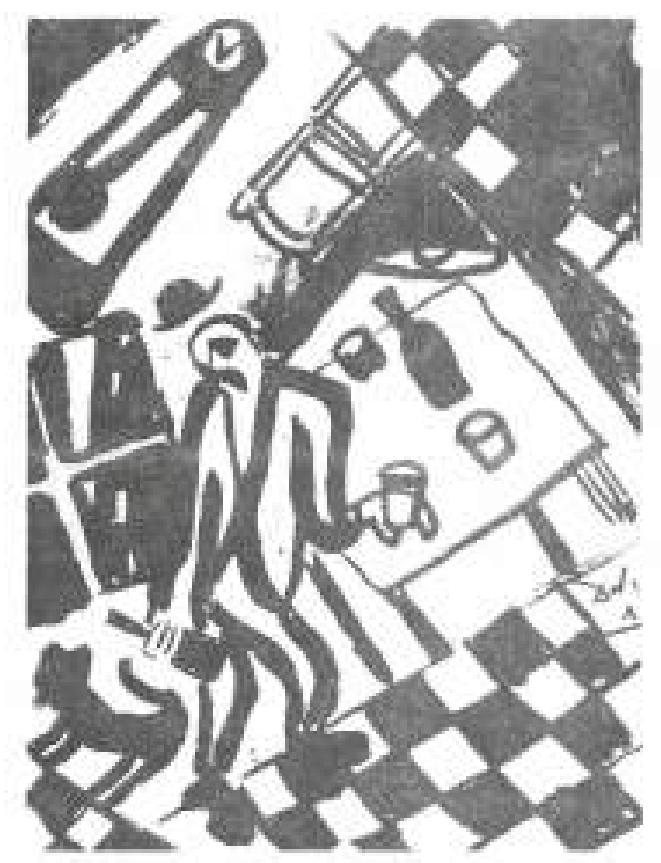

Grupo de Investiga Ición en Ética JOSE HOOVER VANEGAS

Filósofo

LUZ GUIOMAR MALDONADO

Filósofa

LUIS HORACIO FRANCO

MARIA HELENA OROZCO

Psicóloga

Universidad Autónoma de Manizales

\section{DESCRIPCIÓN DEL PROBLEMA}

El concepto de corrupción está más cerca de los fines y medios colectivos de un país, en tanto la violación de los derechos y deberes de los ciudadanos en todos los regímenes, ya sea democrático. socialista o cualquier otro sistema político; la comupción más que la alteración de las leyes juridicas por medio de una acción individual o colectiva, conforma uno de los atentados contra la humanidad como tal y contra el fin último de todo ser inmerso en una sociedad, en otras palabras, la comupción no soloes un problema jurídico sino, quizá con mayor importancia, es un problema ético.

Cada vez que hablamos de corrupción siempre pensamos en una acción que está en contra de los intereses de la sociedad en lo referente al bienes. tar humano y en favor, generalmente, de intereses particulares y de poderes económicos del ejecu- 
tante de la acción o de quien la mandó a ejecutar, por esto la comupción lleva implicito un status de poder, y por ello se relaciona, casi siempre, con to polí tico, sin olvidar que también $5 \mathrm{e}$ da en lo privado.

Sin embargo, si analizamos el concepto mismo de corrupción. en tanto seres humanos que pretenden ganar beneficios a costa del trabajo, el honor, la dignidad. y ain, la liberud de los otros, ya no hay que pensar tanto en to político, sino en la estadia del hombre en el mundo de acuerdo con los valores morales, esto es, en tanto seres más humanos.

En esta medida, la comupción es un problema de humanidad y no s6lo de la sociedad jurídica como tal, por lo tanto exige un tratamiento a la luz de conceptos morales taies como: normas éticas. fines individuales y colectivos. virtudes y valores, esto es, es posible develar la comupción a la luz de la libertad humana para encontrar asi los parámetros que rigen cada uno de estos conceptos $y$, en nuesiro caso, la autonomía como uno de los valores que se desprende de la tibertad.

Este trabajo sólo pretende poner en evidencia la incidencia de la comupción dentro de la evolución racional y argumentada de las personas de acterdo con los fines colectivos e individuales que toda sociedad debe perseguir, en aras de buscar la convivencia pacifica y el desarrollo normal de las personas con base en unos parámetros éticos minimos y máximos, que contribuyan al mejor vivir de la comunidad en general.

El trabajo, entonces, estará dividido en tres partes: primero, intentaremos mostrar la ética y la monal como un problema real de Is vida cotidiana. Segundo, la corrupción y su incidencia en el ser del bombre. $Y$ tercero, intentaremos articular el concepto de autonomia con el de corrupción y sus implicaciones.

\section{L.A ETICA Y LA MORAL COMO PROBLEMAS REA. LES}

Entendemos la ética ea el sentido del êthos griego, el espacio en relación con el hacer mismo del hombre, ¿quế seria del hombre sin un lugar para co-habitar? y esta misma residencia es la que le permite vivirse como un acto. Ef morar, por consiguiente, es lo que el hombre hace de la tierra que habita, es la relación hombre hogar, el morar del hombre se puede entender como et significado privado de donde surgen todas las vivencias y, por consiguiente, el fundamento de todos los comportamientos, asi lo afirma Heidegger

vrowysumfirca exwacia lugar del morar. La palabra nombra la soaa abierta dunde el hombre. 
mora. La aberturad de su estancia hace aparecer to que atviene a la exencia del hombre y wiviendo asf se detiene en sa cercunía. La exlancia del hovibre conviene y conserva la verida de to que pertenece al howbre ensu escracia.'

La ética como carácter pensado nos transporta a su significación más pristina, esto es, el lugar en donde yo mismo me habito, la zona abierta donde el hombre mora. La morada del hombre es representada por la pre-dación de la realidad antes de ajustar un acto, esto es, los seres racionales tiene ia capacidad de adelantarse a las consecuencias de los actos, de idear fantasmagóricamente una reflexión en torno a las consecuencias de un ajustamiento, es decir, el hombre posee la capacidad de hacerse a sí mismo en su propio yo como constructor de nuevas realidades, y esto sólo se logra mediante el pensamiento, por ello la ética es el carácter pensado, el lugar que el hombre se habita antes del ajus tamiento.

Podemos afirmar que las acciones van pre-cedidas de una decisión la cual debe ser fundamentada en la libertad, entendida ésta como la autodeterminación de la voluntad; la libertad es la base de

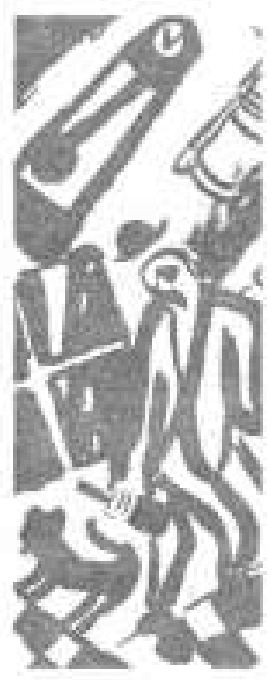

toda moralidad, es ia facultad de decidir en favor de lo correcto y en contra de lo incorrecto. La libertad es la facultad de la razón que le permite al ser humano su misma humanidad. Sin embargo, la corrupción es un acto en contra de la libertad. puesto que la alteración de las reglas por un individuo en beneficio propio implica una coacción de la elección de los otros. La corrupción interviene en el cjercicio de la libertad y por tanto en el desarroIlo moral.

La mayoria de las veces consideramos que los problemas morales son problemas abstractos, inmersos en teorias inalcanzables, y producto de especulaciones que nada tiene que ver con la realidad, sin embargo, como intentaremos mostrarlo, antes de iniciar la reflexión sobre la corrupción y la autonomia, los valores y todos los conceptos implícitos en ella, los problemas morales son viscerales, esto es. son conflictos en donde las personas están imbricadas desde su esencia como seres corporales en el mundo de vida.

De acuerdo con esta posición podemos afirmar que toda expresión es una actuación moral. es decir, que el movimiento corporal lleva impresa una intencionalidad de acuerdo con unas reglas éticas impuestas por la sociedad y, en determinados casos modificadas por los individuos. en forma de hábitos que moidean el ajustamiento mismo. El cuerpocomo movimiento comunicativo, es dado como significación moral; la expresión del cuerpo. en este caso, es una manifestación de valor en el mundo de vida; ¿en qué otro lugat puede habitar lo moral si no es en el cuerpo mismo?

¿Qué es, paes, la inmoratidad o la moralidad" Evidentementa, es ana expessiva respuesta somatica a algo existente en el entorno del individuo. Docir que la conducta de otro hombere "es inmonal", es decir, mas bien. qae usted experimenta us deter. minado conjunto de eventos neuromasculanes en respuesta a su condicita, i Quc es, entorkes. y dónde esta la "inmorulidad"t? Sin discrusión, el "dónde" es el cuerpo y of "que" ex una acti- 
vidad neurumuscular autónoma del cuerpo?

La moral es la praxis misma del hacer del hombre $y$ este hacer sólo se muestra a través del cuerpo vivido como una donación gestual al mundo de vida y tal expresión es producto del saberse mismo del hombre, del lugar que uno mismo habita. La expresión corporal es un comunicar en el mundo y en el comunicar se da el movimiento significativo y moral, en la medida en que se rechaza o se admite lo expresado por los otros, de stierte que la moral es una inter-corporalidad en el hacer diario de los hombres.

Aquí surge una cuestión bien interesante: ¿existe concordancia entre la conciencia de querer mostrar de acuerdo con una expresión o un gesto hacia otros y lo mostrado por el cuerpo? Esto es. ¿siempre qué se hace un gesto de desprecio corporal significa que se está despreciando?. Esto to podemos demostrar diciendo que el hombre no sólo se sabe a sí mismo, sino que también se puede mostrar a otros como quisiera que ellos lo supieran, y aún más, un hombre puede mostrarse desde lo que el cree que es y no desde lo que es.
En este sentido aparece el término moral de sinceridad, el cual es definido como una virtud, (un hábito bueno); también aparece el término persona, del latín personata el cual es traducido como enmascarada, persona desde su etimología significa máscara. lo mostrado por el cuerpo cuando se siente algo diferente. es decir la disociación entre la consciencia de lo que se quiere mostrar y lo mostrado. ¿Acaso el ser sociable del hombre no es, en mucho sentido, un tener que estar con los otros? Aquí aparece el concepto de deber, entendido como la aceptación corporal del alter ego.

El cuerpo, entonces, es el mostrario escénico de las interacciones morales. de acuerdo con la ruptura o concordancia entre to que se muestra gestuaimente y la consciencia al mostrarlo: los sentimientos morales, por ejemplo: la vergüenza, la culpa y la indignación, son

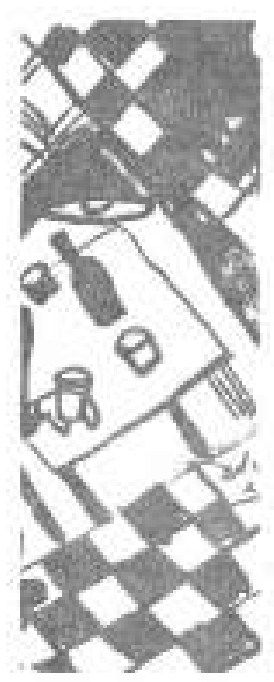

desciframientos corporales de lo que la persona pretende y lo que muestra corporalmentc, por esto nos dice Rawls, en la Teoria de la Justicia:

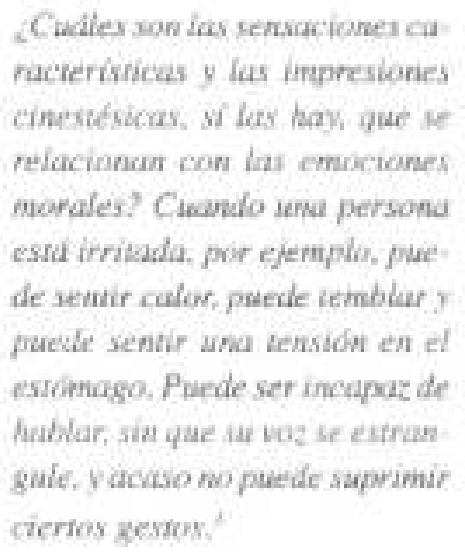

De acuerdo con esto podemos advertir que la moral, cual elongación corporal en el mundo de vida, para los otros y desde los otros, encierra conceptos éticos, tales como voluntad, fia autoderminación de las actos), el cuerpo en tanto vehículo de la conciencia, del yo puro; la sinceridad como la concordancia entre to que se muestra gestualmente y to que se piensa, la personalidad como la solidificación del carácter, como el modo de ser de cada uno de los individuos y enmascarado por el cuerpo. los sentimientos morales y la labor del cuerpo en tanto su exteriorizaciōn. 
En este sentido, podemos afirmar que el cuerpo es la fachada del pneuma (el alma), de la concieneia de lo que el hombre mismo se sabe, Esto implica que el cuerpo vivo es el lugar que permite a los individuos hacerse como seres sociales y de acuerdo con una reglas éticas especificas, en tanto unos acuerdos tácitos o explicitos. por esto, el cuerpo además de ser el punto eje en donde se encuentran todas las experiencias es quien propicia las vivencias mismas: "El cuerpo es el vehiculo del ser del mundo "d

La dialéctica entre lo donado del cuerpo al mundo de vida y la conciencia del mostrar, de los deseos, de los sentimientos y de los valores más intimos del pneuma hurnano se mimetizan en el gesto corporal, por esto afirma Merleau-Ponty que: " $E l$ cuerpo se ha vuelto el escondrijo de la vida"s

Bajo estos criterios éticos, podemos afirmar que la comupción altera el hábitat de todo ser humano, genera una ruptura en la formación de los individuos como un item en las impresiones de los sujetos $y$, lo que es peor en el imaginario colectivo de toda una comunidad, de tal forma, que pasa a constituir la base de un actuar normal al interior de una colectividad, en esta medida todo acto corrupto es un elemento que degrada no sólo a quien lo ejecuta sino a todo el hacer moral de la humanidad.
LA CORRUPCION: UN PROBLEMA DE HUMANIDAD

La tenencia o lo propio en el sentido de la acumulación de bienes, de funciones o de fuerza de trabajo per cápita, en general, parece ser una disposición caracteristica de todos los hombres $y$. máxime dentro de los sistemas económicos capitalistas, la evidencia nos es dada por las relaciones de las personas en la vida cotidiana: el amigo más interesante es el que tiene mejor carro o mejor posición económica, o en otros casos el más inteligente, obsérvese que en estos casos la amistad no es una relación entre dos personas, sino entre dos medios, dos útiles, en donde siempre se está intentado sacar el mejor partido, esta premisa la sustenta la observación de la vida diaria, el hombre vale por lo que tiene, no por to que es.

Teniendo en cuenta que la tenencia es un medio y el ser de los individuos es el fin, las manifestaciones más corrientes de los hombres están determinadas por los medios que utilizan para mostrarse como lo que son, esto significa, que se ha confundido to mostrado por las personas desde su condición como fin, por los medios mostrados en tanto tenencias, podemos afirmar que, uno de los imaginarios colectivos más importantes de esta época es esconder el ser detrás del tener, en otras palabras, los medios de *...podemos afirmar que la corrupción altera el hábitat de todo ser humano, genera una ruptura en la formación de los individuos como un item en las impresiones de los sujetos $y$, lo que es peor en el imaginario colectivo de toda una comunidad...»

\section{-48. Universidad Autonoma de Manizales}


la subsistencia han mimetizado el ser mismo de cada persona.

De acuerdo con esto podemos decir, en una primera instancia, que la comupción es la büsqueda de medios por encima de los fines o dicho de otra forma, la bús: queda del tener por encima del ser, es la disposición de algunas voluntades de luchar por el tener sin importar los seres humanos en sí mismos, tanto individuales, como colectivos, como instituciones o como paises. La corrupción, en este sentido, es la ruptura del desarrollo humano en tanto la bús: queda de la justicia, ejemplos de este tipo los tenemos en abundancia: después de la Constitución Colombiana del 91 no son pocos los políticos que han sido acusados por corrupción.

A hora bien, ¿̇cuál es, por decirio de alguna forma, la esencia del tener? La posesión procura en quien posee la sitisfacción de necesidades tanto básicas como de comodidad y, aún, de lujo, pero también procura facultades de reconocimiento social. En cualquiera de los dos casos la tenencia brinda poder. $\mathrm{E}$ dominio de un individuo estí determinado por la forma de poder que ejerza sobre otros, en esta medida podemos enriquecer nuestra primera aproximación al concepto de corrupción afirmando que ésta es la utilización de medios ilegales en la búsqueda del poder.

A la estructura del manejo del poder, o al ejercicio del poder, es a lo que se ha llamado política, quizá por esto, algunas veces, homologamos la corrupción con la nombrada política, aunque en realidad, la política no es corrupta en sí misma, lo corrupto es la politiquería y mas precisamente los politiqueros.

La política, como manejo del poder, nos da la idea de reglas, las cuales son, en últimas la urdimbre de toda estructura social. El com-portarse de los hombres con sus semejantes debe estar sometido a los fines individuales y éstos a sa vez a los fines de la sociedad regidos por el Estado, pero los seres humanos gozan de libertad, capacidad de decidir, en favor o en contra de los otros, por esto toda sociedad debe estar sometida a unos preceptos que regulen su propio comportamiento en favor de la colectividad, tales preceptos no son otros más que las reglas.

El cumplimiento o el incumplimiento de las reglas define el fin tanto de los individuos como de las comunidades y de acuerdo con él se puede derivar su característica en cuanto al ser de las personas o el tener de las mismas con lo cual encontramos el ejercicio del poder de acuerdo con la acumulación, o el desarrollo humano en favor de la comunidad. Aquí encontramos los elementos para acercarnos aún más a lo que se ha denominado como corrupción: ella consiste en la violación de las reglas de una «...uno de los imaginarios colectivos más importantes de esta época es esconder el ser detrás del tener, en otras palabras, los medios de la subsistencia han mimetizado el ser mismo de cada persona.s 
comunidad para el beneficio propio, esto si tenemos en cuenta que las reglas de la comunidad en donde se actue sean correctas, o sea, que se den en pos del bienestar humano, porque puede haber reglas en comunidades que sean problemáticas en este sentido.

De acuerdo con este panorama podemos concluir hasti aquí que la corrupción siendo un problema del tener, del uso de los medios para llegar al poder por encima de la reglas de una comunidad, es un problema de humanidad; puesto que, ni el tener, ni los medios, ni la economía ni la práctica son corruptos en sí mis. mos, la corrupción es un proble. ma de actitud, de voluntad humana.

En este sentido lo más importante, a nuestro modo de ver, no es lo que se gana a nivel de tenencia o nivel de poder, ni tampoco lo que se pierde en cuanto medios de subsistencia, sino lo que se gana en cuanto ser racional o lo que se pierde en cuanto ser intencional, en otras palabras el problema de la corrupción es un problema ético, antes que un conflicto jurídico.
Sin embargo de aqui podemos inferir una pregunta: ¿qué es más importante al interior de una sociedad, castigar a los corruptos o formar argumentativamente a los seres de una comunidad en tanto personas con libertad y por tanto con autonomia?. Esta pregunta no amerita una respuesta explicita puesto que su obviedad es de sentido comuin, pero la respuesta es nuestro argumento para reforzar la tesis que defendemos: la comupción es un problema de humanidad, es un problema moral.

Si estamos de acuerdo con esta posición, hay que afirmar que las acciones corruptas no sólo lo son por el beneficio económico de unos pocos y la negación ilegal del mismo a otros. La alteración de las reglas implica toda una forma de habitualidad que en úttimas repercute en el desarrollo humano en cuanto a lo moral y afincado en la somaticidad, como lo que legitima la individualidad, esto es, altera la libre expresión del hacer del hombre en tanto ser libre, es decir, la corrupción tiene repercusiones en Ia racionalidad moral de tal forma que estas alteraciones pueden llegar a ser asumidas por una comunidad como actividades normales, y esto implica una formalización de la comupción al interior de una comunidad, lo cual es lo peor que puede pasar a un individuo, pues. to que estas prácticas son más propensas a la destrucción de la humanidad

En esta medida, la corrupción no sólo se manifiesta en donde haya intereses economicos o políticos, sino en donde esté involucrada la dignidad de cualquier ser humano, puesto que la corrupción lo es en tanto lleve implicita una violación contra el ser mismo de los individuos, de acuerdo con esto. corrupción es, desde el engaño o la mentira en cuanto al conocimiento, un docente que miente a sus estudiantes en favor de su propia imagen, hasta ef fraude de un político.

Si bien la corrupción lleva implicitos unos intereses individuales de acuerdo con la ruptura de unas reglas al interior de una comunidad, es de fundamental importancia pensar el concepto en cuestión en tanto la integridad moral del hombre, por ello en lo que sigue nos instalaremos en la autonomia y la incidencias de la corrupción en ella.

\section{LAAUTONOMIA Y LA CORRUPCIÓN}

Las reglas en tanto limites que exhortan un acto y prohiben orros son determinadas como correc- 
tas, si contribuyen al bienestar humano $y$ a la felicidad individual sin oponerse a los fines de los demás o incorrectas si obstaculizan el bienestar humano. En este sentido podemos entender el valor de la autonomía, como el interés de un individuo por las reglas correctas de acuerdo a su propio fin. La autonomía es la capacidad de la voluntad individual de vivir de acuerdo a sus propias reglas sin violar las normas de unis colectividad.

La autonomía como un valor que se deriva de la libertad posee tres características fundamentales como minimo, primero la auten: ticidad, esta cualidad de la autonomia implica una cierta identificación con la elección de las reglas que van a regir el comportamiento del individuo, pero tam. bién ln elaboración de nuevas reglas en tanto no viole los dere. chos y los deberes de los otros. La autenticidad es una cualidad de la autonomia puesto que debe cumplir estos parámetros y sin embargo, tanto en su elección como en su formación tiene ele. mentos creativos. No obstante, esta autenticidad se ve debilitada cuando una alteración de las reglas morales interviene en su ejecución.

Segundo, La reflexión racional, esta característica denota en los seres morales toda la facultad pre-dada, el análisis de las impresiones de acuerdo con el fin de la acción misma. La racionalidad implica poner en cuestión las consecuencias de la acción de acuerdo con la conciencia del agente ejecutante de fos actos en tanto la integridad de la otredad. La autonomía en este sentido implica la inclinación por las reglas y principios correctos en favor del orden justo de una comunidad, como lo afirma John Rawis:

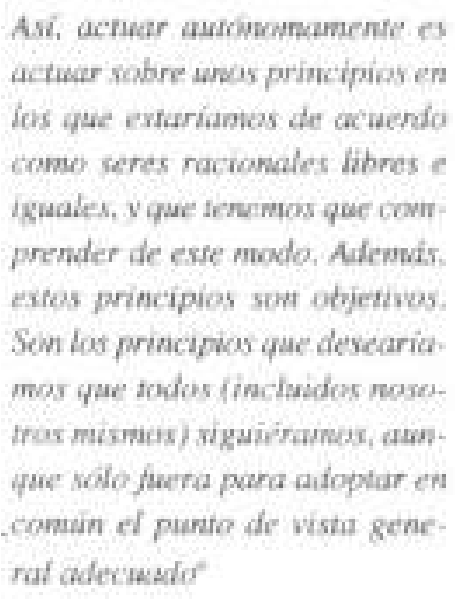

Ahora bien, la autonomia lleva en sí misma la elección racional de lo justo, pero ello implica que existan otras elecciones: lo injusto, lo imparcial, o al contrario la elección de la parcialidad implica que exista algo injusto. La corrupción en este sentido, al condicionar la libertad puede implicar la ausencia de elección en tal medida que la única elección de acción de un individuo sea una acción heteronoma. por ejemplo: cuando un individuo no tiene para suplir sus necesidades primarias y las de su familia, es probable que elija robar, puesto que lo contrario significaria una disminución en la calidad de vida. La corrupción en algunos, y no pocos casos, implica la supresión de la autonomía en tanto reflexión racional.

\section{Tercero: la fuerza de voluntad.} Para que esta característica de la autonomia se cumpla es necesaria la cualidad antes expuesta en el ejercicio de la libertad. Aunque la voluntad, como la fuerza del deseo por ejecutar unos actos y no ejecutar otros. lleva implicito un carácter psicológico. también podemos verlo como la consolidación del carácter por medio de los hábitos buenos que en últimas se convierten en costumbres, por eso la personalidad es lo que se ha conquistado en las vivencias mismas, como to afima Aranguren: "... El caracler constituye una imprevión de rasgos en la persona misma: el carácter es la personalidad que hemos conquistado, a través de la vida, lo que hemos hecho de nosotras mismos vivienda"? 
La fuerza de voluntad depende. en muchos casos. de la habitualidad de la personalidad de los individuos y aunque ella constituye la resistencia del individuo por lo fácil, lo agradable, en tanto incorrecto, debemos entender que no todo lo fácil y lo agradable es incorrecto, en este caso es lo que esté en contra de la humanidad. la fuerza de voluntad consiste en la capacidad de los seres humanos de inclinarse por las reglas que contribuyan al bienestar de la humanidad.

La autonomía y en general todos los valores son preferencias que se tejen culturalmente al interior de un época, y se legitiman en la medida en que se ejerzan por los individuos, esto es, los valores como la valía de las acciones correctas cobran más importancia para un determinado momento histórico en tanto sea más reconocido por las personas al interior de esa cultura; no existen los valores sin la valoración, nos dice Frondizi ${ }^{\mathrm{B}}$

Los valores son cualidades reales de las acciones, ellos cobran independencia de los sujetos valoradores, pero dependen del valorar, to cual significa que sí bien las personas legitiman las valencias o se interesan por las cualidades buenas de las acciones, ellas cobran tal independencia que, en últimas pueden modificar al sujeto valorante, se im- ponea en una cultura y determinan la conducta de los individuos al interior de la misma.

Las normas de una sociedad necesitan tener por base un sistema de valores, éste es, quien en últimas, en tanto preferencia implica la ejecución de la norma o et incumplimiento de la misma. pero las normas deben partir de la necesidad cultural y no deben ser una imposición arbitraria; en otras palabras, el valorar la norma posibilita la labor de la misma en una sociedad. En esta medida, la valoración de los valores determina el grado de justicia de una comunidad, en la medida en que tal valoración sea producto de las necesidades culturales y no se opongan al bienestar de la comunidad, en tanto que persigan un fin correcto.

La corrupción conforma una de las peores enfermedades de nuestra cultura porque ella implica una nueva imposición cultural de valores, no sólo con relación a la autonomía sino con relación a las valencias de nuestra comunidad, en tanto valoración negativa porque eleva el valor de la tenencia por encima del valor del ser, el cual en últimas ha cobrado precio, esto es una cultura debe valorarse por la determinación del ser por encima del tener pero la corrupción implica la determinación del ser por debajo del tener.
- Ponencia leuda en el tercer foro interuniversitario de Etica y Corrupción el 23 de Septiembre de 2000

' HEIDEGGER. Martin. Carta sobre ei Humanismo Madnd. Tauros. 1960 . p. 56.

${ }^{2}$ HANNA Thomas La Retelión de las Cuerpos. Barcelonk. Plaza y Janes S.A. 1972 , p. 34

'RAWLS, John. Tereia de fa Justicia. Méxion: F.C.E segunda edación. 1995 . ㅍ. 434

MERLEAU-PONTY. Maurice Feanmenologia de las Ciencias aumanas Buenos Aires. segunda Edición. Novi. 1969-p: 100

"Thid. p: I8L.

- Estas carncteristicas de la autonomía son tomadas de Stanley Bean, en Condición de la Ausconomia, citado por R. S. Piter. Desarrollo Moral s Edacación Moral. Mérico F.C.E.

"RAWLS, JOHN, Terria de la Jesticia, Mevicox FCE segunda Edicón. 1995, if. 467

ARANGUREN, Jose Lais L. Ehea Barcelona: Altaya 1994 \& 23.

* FRondizl. Risieri. Pensamieato Axiologico, Cali, Colombia: Universidad del valle 1993, p 12 